\title{
EL PLANETA HEMBRA DE GABRIELA BUSTELO: DESCIFRANDO UNA IDENTIDAD POSHUMANA
}

\author{
GABRIELA BUSTELO'S PLANET WOMAN: \\ DECIFERING POSTHUMAN IDENTITY
}

\author{
Juan Carlos Martín \\ Stonehill College
}

\section{RESUMEN}

Algunos críticos han afirmado que el poshumanismo tanto precede como sigue al humanismo. Muchos de ellos concuerdan en que el poshumanismo ha de concebirse como un paradigma informado por avances tecnocientíficos que sirven para reconfigurar nuestra forma de definir lo humano. Planeta hembra, de Gabriela Bustelo, nos ofrece un buen ejemplo de la confluencia poshumana de biología y máquina en cuanto a las dimensiones de poder, sexo, y género se refiere.

Palabras clave: Poshumanismo, cyborg, tecnociencia, manipulación genética, género.

\begin{abstract}
Some critics have argued that Posthumanism both precedes but also follows Humanism. Many of those critics also agree that Posthumanism must be conceived as a paradigm driven and sustained by exponential technological and scientific developments and their impact in reconfiguring normative conceptions concerning our understanding of what it means to be human. Gabriela Bustelo's Female Planet (Planeta hembra) offers a valid approach to exploring notions of such a posthuman condition in which the merging of biology and machine redesigns notions of human identity, drives human desire and reshapes attitudes regarding issues of power, sex and gender struggles.
\end{abstract}

Keywords: Posthumanism, cyborg, technoscience, genetic manipulation, gender. 
Las últimas décadas del siglo XX y los primeros lustros del siglo XXI han sido testigos de un avance tecnológico acelerado, así como de trascendentales hallazgos científicos, especialmente en los campos de la ingeniería genética y la biología molecular. ${ }^{1}$ Estos y otros hallazgos tecnocientíficos permiten hoy en día manipular de manera artificial ciertos procesos evolutivos naturales que antaño determinaban las nociones básicas en torno a lo que significaba ser humano. Algunos críticos e investigadores entrevén una relación inherente entre el avance tecnocientífico $-y$ su impacto directo en la configuración de la identidad humana- y el establecimiento de una condición poshumana ineludible. ${ }^{2}$ Hablando de la necesidad de revisar el paradigma humanista, el autor Robert Pepperell ${ }^{3}$ afirma que el concepto de poshumanidad marca el final del humanismo, cuestiona la configuración tradicional de la humanidad y alude a una creciente simbiosis entre lo artificial y lo biológico, al punto de problematizar la distinción entre ambos fenómenos (iv). ${ }^{4}$ Katherine Hayles advierte que muchos autores conciben la llegada de una poshumanidad con cierto recelo, e incluso miedo, mientras que otros celebran con entusiasmo las posibilidades que podría llegar a ofrecer una condición poshumana en la configuración de un sujeto contemporáneo.

1 Véase por ejemplo la creación de la primera célula viva con ADN sintético capaz de emular las funciones de una célula biológica. https://www.newscientist.com/article/dn18942-immaculate-creation-birth-of-the-first-synthetic-cell/.

2 A finales de los años setenta lhab Hassan vislumbraba ya un cambio paradigmático en la concepción filosófica de la naturaleza humana y la configuración de un sujeto racional cartesiano: "We need first to understand that the human form - including human desire and all its external representations - may be changing radically, and thus must be re-visioned. We need to understand that five hundred years of humanism may be coming to an end as humanism transforms itself into something that we must helplessly call post-humanism" (212).

3 Véase "The Posthuman Manifesto" (Kritikos, Vol. 2, Febrero 2005). http://intertheory.org/ pepperell.htm. 
El miedo, argumenta Hayles, lo provoca la posibilidad de que las inteligencias artificiales generadas en una era poshumana puedan llegar a precipitar el desplazamiento del ser humano como forma de vida dominante en el planeta (283). ${ }^{5}$ Por el contrario, continúa Hayles, aquellos que reciben con entusiasmo la poshumanidad, vislumbran dentro de este paradigma la posibilidad de generar nuevos planteamientos críticos para explorar con mayor amplitud la configuración de un sujeto humano presente (285). ${ }^{6}$ Al igual que Hayles, otras voces expertas en torno a la poshumanidad, como Judith Halberstam e Ira Livingston, o el propio Neil Badmington, interpretan la llegada de una condición poshumana como una oportunidad "to engage discursive and bodily configurations that displace the human, humanism, and the humanities" (Halberstam y Livingston vii). Estos y otros críticos coinciden además en que la poshumanidad se precipita de manera acelerada en las últimas décadas al producirse avances tecnológicos y científicos sin precedentes que cuestionan seriamente la validez de ciertas divisiones binarias humanistas: hombre/máquina, orgánico/artificial o virtual/real, entre otras. ${ }^{7}$

El debate sobre esta condición poshumana se ha abordado con bastante frecuencia desde varias disciplinas, siendo la biotecnológica y la tecnocientífica las más desarrolladas y las que mayor interés suscitan, sobre todo a nivel de la teoría crítica, la literatura y el cine. En este sentido, la novela de la autora madrileña Gabriela Bustelo, Planeta hembra (2001), sirve como botón de muestra para llevar a cabo un acercamiento válido desde

4 En The Posthuman Condition: Consciousness Beyond the Brain (2003), Robert Pepperell indaga de manera interdisciplinaria en la configuración y establecimiento de un paradigma poshumano. El autor analiza el concepto de la poshumanidad explorando, entre otras cosas, la manera en la que las inteligencias artificiales, la nanotecnología, las manipulaciones genéticas o la vida artificial desafían la configuración identitaria humanista del individuo.

5 En Our Posthuman Future: Consequences of the Biotechnology Revolution (2002), el politólogo Francis Fukuyama explora críticamente la poshumanidad y su impacto biotecnológico y científico en el devenir de una sociedad humana futura.

6 En How We Became Posthuman: Virtual Bodies in Cybernetics, Literature, and Informatics (1999), Hayles aborda el concepto de la poshumanidad relacionando tres historias: "how information lost its body [...] how the cyborg was created as a technological artifact and cultural icon [...] how a historically specific construction called the human is giving away to a different construction called the posthuman" (Hayles 1999, 2).

7 Paula Rabinowitz sugiere que los cuerpos poshumanos "are bodies living outside national, sexual, economic borders. They exceed and override borders by turning bodies into acts and actions into representations" (98). 
la ficción al concepto de la poshumanidad y a la construcción de una sociedad poshumana en la que la convergencia entre lo biológico y lo artificial moldea y condiciona de manera incuestionable la identidad, el deseo y el devenir de una raza humana futura. Partiendo de la base de que el impacto tecnocientífico y biotecnológico está cambiando de manera acelerada el perfil de una sociedad contemporánea, y tomando como marco de análisis literario esta novela, este estudio examina las siguientes cuestiones:

En primer lugar, plantea cómo dentro de la ficción los vertiginosos avances biotecnológicos y tecnocientíficos precipitan la construcción de organismos cibernéticos que reconfiguran y cuestionan la identidad de un sujeto dentro de un paradigma humanista tradicional. Asimismo, este artículo examina brevemente cómo en la ficción el monopolio y la politización biotecnológica y tecnocientífica pueden influir de manera negativa en la construcción de una sociedad poshumana futura. Un tercer cometido explora cómo la experimentación y manipulación tecno-sexual con y en el cuerpo femenino, permiten concebir la posibilidad de un paradigma genérico-sexual alternativo al perpetuado históricamente por un patriarcado humanista. Vale la pena señalar que en Planeta hembra las cuestiones en torno a la poshumanidad se abordan teniendo en cuenta el tono paródico que imprime la autora al aproximarse al género de la ciencia ficción y, más concretamente, a la ciencia ficción feminista; desplegando en su narrativa supuestos distópicos propios del género. Pero antes de pasar al análisis de la novela, resulta necesario examinar con mayor detalle algunos factores esenciales que en las últimas décadas han generado una respuesta crítica al fenómeno de la poshumanidad.

En relación al acelerado avance tecnocientífico y su impacto en el ser humano, Elaine Graham afirma que los límites entre lo biológico y lo artificial se borran cada vez más debido a los avances en las tecnologías reproductivas, la clonación, las modificaciones genéticas y la tecnologización del cuerpo humano con fines terapéuticos (3). Según la autora, estos avances biotecnológicos y tecnocientíficos, influidos también por las tecnologías digitales, cuestionan seriamente "the ontological purity according to which Western society has defined what is normatively human" (5). La supuesta contaminación ontológica que insinúa Graham, subraya una creciente simbiosis orgánico-artificial y suscita múltiples polémicas, y como afirmaba más arriba Hayles, evoca también varios placeres y temores. Hoy en día, resulta una práctica común el uso de tecnologías innovadoras aplicadas al cuerpo para mantener y mejorar la calidad de vida de personas afectadas por una enfermedad o un accidente. Sirvan como ilustración de estas tecnologías, las prótesis convencionales, los marcapasos o los corazones artificiales que reemplazan los biológicos; o incluso el uso de 
prótesis mucho más avanzadas, como las retinas artificiales, con una capacidad sorprendente de enviar señales visuales del nervio óptico al cerebro.

En última instancia, la manipulación tecnológica y científica del cuerpo humano ahonda de manera certera en la configuración ontológica de una identidad asociada directamente a la poshumanidad, el cyborg: "a self-regulating organism that combines the natural and the artificial together in one system" (Hables Gray 2). El neologismo cyborg -término complejo y ambiguo, es una contracción de las palabras cibernético y organismofue acuñado en 1960 por los científicos Manfred Clynes y Nathan Kline para referirse a una manipulación tecnológica del cuerpo que permitiera al hombre la exploración del espacio exterior. ${ }^{8}$ Aunque el género literario de la ciencia ficción y el cine han tergiversado el significado original del término cyborg, ${ }^{9}$ hoy en día la entidad cyborg es una realidad social y tecnológica irrefutable, como plantea Chris Hables Gray en su sugerente volumen Cyborg Citizen (2001).

Habría que puntualizar que la identidad cyborg, como sugiere Hables Gray, no hay que concebirla necesariamente como la unión de mamífero y sistema cibernético, puesto que "any organism/system that mixes the evolved and the made, the living and the inanimate, is technically a cyborg" (2). Del mismo modo, Donna Haraway vislumbra al cyborg como un híbrido, pero también como "a creature of social reality as well as a creature of fiction" (149), en el que se llegan a inscribir cambios políticos, socio-culturales y tecnocientíficos que condicionan y moldean la formación de una identidad poshumana. ${ }^{10}$ En este sentido, señala Neil Badmington, el cyborg supone para Haraway una poderosa metáfora para abordar la crisis del humanismo "[and] many of the assumptions of humanist discour$\mathrm{se}^{\prime \prime}(88)$. Por otro lado, dentro de un paradigma poshumanista, a este organismo cibernético - fruto de la medicina biotecnológica, de la metáfora o incluso de la ficción literaria y cinemática - se le unen otras identidades que se generan virtualmente en una realidad cotidiana, y que dan origen a un sujeto poshumano incorpóreo: "a technology, a screen, a projected

8 Para una explicación más detallada del concepto original del término cyborg, véase Manfred Clynes y Nathan Kline "Cyborgs and Space" en The Cyborg Handbook (1995).

9 En la cultura popular destacan varias versiones de organismos cibernéticos con rasgos y comportamientos humanos como los que exponen los replicantes de "Blade Runner", el propio "Robocop" o una versión del cyborg a la inversa, el "Terminator": un ente con apariencia y una conducta cognitiva humana, pero dotado de un cerebro artificial gobernado por un microchip.

10 Véase el capítulo ocho en Simians, Cyborgs, and Women, "A Cyborg Manifesto: Science, Technology, and Socialist-Feminism in the Late Twentieth Century". 
image" (Halberstam y Livingston 3). Además, arguyen Halberstam y Livingston, no hay que obviar la existencia de otras identidades poshumanas que se generan, por ejemplo, al concebir un cuerpo humano "under the sign of AIDS, a contaminated body, a deadly body, a techno-body [...] The human body itself is no longer part of 'the family of man' but of a zoo of posthumanities" (lbíd. 3). Si bien es verdad que no todo sujeto poshumano es necesariamente un cyborg, un cuerpo contaminado o una tecnología digital, las reflexiones críticas anteriores ratifican que el sujeto de la era poshumana es un ente que está sufriendo una transformación notable. En última instancia, lo que parece evidente es que la consolidación de un paradigma poshumano, respaldado directa o indirectamente por un impacto tecnocientífico y biotecnológico considerable, precipita de manera incuestionable el éxodo de una identidad marcada por el humanismo, la del homo sapiens, y permite además concebir y reconocer la emergencia paulatina de otra identidad, a saber, la del homo cyberneticus:

El homo cyberneticus deja atrás al hombre centro de la creación como afirmaban los pensadores medievales y también al hombre dueño de lo existente que postulaba el pensamiento ilustrado, el homo cyberneticus se convierte en el creador de naturaleza, quiere convertirse en el diseñador de su propio proceso evolutivo, busca un darwinismo dirigido. ${ }^{11}$

\section{En torno a Planeta hembra}

Junto a escritores coetáneos como Lucía Etxebarría, José Ángel Mañas - Ray Loriga, todos ellos autores de una narrativa neorrealista, Gabriela Bustelo es uno de los exponentes femeninos más notables del grupo de narradores de la llamada generación X, muy de moda en la década de los noventa. Esta generación de escritores centra la temática de su narrativa en torno a una juventud española marcada por el abuso del sexo, la música rock y las nuevas tecnologías, y por un consumo excesivo de drogas y alcohol. A pesar del éxito editorial de algunos de los autores neorrealistas, la crítica literaria ha tendido a desacreditar el valor literario de su narrativa, arguyendo que sus planteamientos estéticos distan mucho de un

11 Véase la versión digital del autor Daniel López Salort "Cyberontología, poshumanismo cibernético y constitución del último hombre". http://www.observacionesfilosoficas. net/cyberontologia.html. 
canon tradicional dentro de las letras peninsulares. ${ }^{12}$ Siguiendo la estela neorrealista, la temática de la primera novela de Bustelo, Veo, veo (1996), se centra en reflejar precisamente los excesos de una juventud española en la urbe madrileña. Pero esta primera novela de Bustelo es además un tributo paródico a la novela de espionaje. En Planeta hembra la autora repite algunos de estos recursos retóricos para parodiar el subgénero de la ciencia ficción y cuestionar de paso los postulados feministas político-sexuales radicales que en la ficción precipitan la instauración del lesbianismo como orientación sexual dominante en el planeta. El recurso paródico permite desvelar además hasta qué punto una "heterotopia lesbiana" (Altisent 89) -es decir, un emplazamiento físico donde se proyecta un deseo utópico lesbiano-, puede conducir a una sociedad carente de memoria histórica, y de valores éticos tradicionales, a su completa destrucción. ${ }^{13}$

Planeta hembra provee una visión distópica de una sociedad poshumana dividida en facciones con convicciones ideológicas, políticas, culturales y sexuales claramente irreconciliables. A un lado de la balanza se posiciona el Partido Mundial de las Hembras, la coalición política XX de mujeres lesbianas. Mediante la ayuda de un control tecnocientífico sin precedentes, el partido XX lucha obsesivamente para erradicar por completo la influencia y poder patriarcal que ejerce el Partido Mundial de los Hombres XY, de orientación sexual supuestamente gay. A esta división binaria se antepone desde la marginalidad un reducido grupo, el Comando $\mathrm{H}$, de clara tendencia heterosexual. Este grupo de insurgentes sobrevive en la clandestinidad de los túneles de un desahuciado metro neoyorkino, para desde allí atentar contra las vidas de las líderes del partido XX.

Además de los ataques terroristas, el Comando $\mathrm{H}$ se dedica a hackear los sistemas de comunicación para colgar en la red global imágenes que exhiben el coito heterosexual, considerado por las mujeres del planeta como lo abyecto y el símbolo por excelencia de un orden patriarcal propio de un legado humanista. Báez, líder destacado del partido XX y directora del Departamento de Erradicación de Grupos Terroristas, y Graf, un líder enigmático del partido de los Hombres $X Y$ aúnan fuerzas para neutralizar los ataques terroristas del Comando $\mathrm{H}$. Después de mantener una relación estrictamente profesional, Báez y Graf comienzan a intimar y su relación

12 Para un aproximación crítica a la narrativa neorrealista, véase por ejemplo, Generation X Rocks: Contemporary Peninsular Fiction, Film and Rock Culture (2007).

13 Para una explicación más detallada del término heterotopia, véase Michel Foucault "Of Other Spaces, Heterotopias." http://foucault.info/documents/heteroTopia/foucault.heteroTopia.en.html. 
acaba desembocando en una ardiente relación heterosexual. Este desliz, prohibido por el partido XX y visionado de manera global a través de la red, se convierte en el detonante de una guerra mundial de sexos que precipita la destrucción del planeta. Milagrosamente, Graf, Báez, Alva, miembro del Comando $\mathrm{H}$, y Dillon, una adolescente inquisitiva del partido XX, sobreviven y ponen rumbo al planeta Andrómeda, restableciendo así lo que parece ser un nuevo orden patriarcal basado en un modelo normativo heterosexual, tan criticado y parodiado en la ficción.

Si en principio la visión que ofrece la novela de una tecnocracia feminista radical resulta un tanto inverosímil, otros temas tratados en la ficción, como pueden ser las relaciones de poder entre sexos o el impacto de la tecnología en la comunicación y la configuración identitaria del individuo, son fenómenos propios y apremiantes de una realidad extra textual contemporánea. En otras palabras, en ningún momento se plantean en la novela escenarios maravillosos o sobrenaturales, ilustrativos de un género fantástico. Asimismo, aunque Planeta remeda encarecidamente un sistema de gobierno feminista futuro, que intenta hacerse con las riendas del poder mundial, la culminación de un paradigma de poder político femenino fuera de la ficción no resulta totalmente descabellado, ni tan siquiera quimérico, si se tiene en cuenta el protagonismo creciente que desde el siglo pasado ostenta la mujer en la esfera política. ${ }^{14}$

\section{IDENTIDAD HÍBRIDA Y ORGANISMO CIBERNÉTICO}

Uno de los temas más controvertidos de la teoría poshumana se centra en la concepción de un sujeto híbrido producto de una simbiosis entre lo orgánico y lo artificial, y la demarcación identitaria que se genera como resultado de esta unión. En Planeta hembra se puede abordar el concepto de identidad cyborg analizando en detalle el comportamiento de algunos de estos organismos cibernéticos. En Planeta destaca la estrecha relación que mantienen la líder Báez y su máquina personalizada Maggie Mae, MM: siglas en honor a la primera ministra "Margaret Thatcher, la única Hembra rescatable del siglo pasado" (15). La primera impresión que se lleva el lector en relación a esta inteligencia artificial es su capacidad cognitiva, que emula sin duda un comportamiento humano, aunque paradójicamente su apariencia de ordenador personal y su voz robotizada contradigan en principio esta imagen. $M M$ se encuentra conectada a la red global, lo que

14 Sirva de ejemplo paradigmático la figura de Margaret Thatcher - un icono político dentro de la ficción para las mujeres o hembras del planeta-, u otras damas de hierro como Hilary Clinton, Angela Merkel, o Cristina Fernández de Kirchner. 
unido a su interacción diaria con Báez y su crecimiento evolutivo dentro del mundo virtual al que se encuentra enganchada, multiplican su capacidad cognitiva, su autoconsciencia y por ende, su autonomía como ente.

La líder Báez, también concebida como organismo cibernético mediante la manipulación genética y la clonación selectiva, ejemplifica el tipo de sujeto cyborg en una sociedad poshumana que debe depender de la tecnología para su gestación, desarrollo y supervivencia. En el caso de Báez y otras mujeres líderes, su condición de organismo cibernético se refleja en las alteraciones genéticas sufridas para cambiar procesos evolutivos y biológicos naturales propios de la especie humana, como puede ser por ejemplo el ciclo menstrual, un fenómeno que para las mujeres del planeta no es más que una "tara primitiva" que la ingeniera genética se ha encargado de corregir (181). Otros avances tecnocientíficos mejoran estas identidades cyborg, controlando procesos biológicos naturales a través de sistemas cibernéticos, con la ayuda de la nanotecnología, que permiten, sin ir más lejos, erradicar un "brote cancerígeno microscópico" implantando en el cuerpo "un chorro de moléculas inteligentes" (52). En este sentido, Báez y otras mujeres del planeta, transformadas claramente por una dependencia tecnológica exagerada, se convierten en sujetos poshumanos que contrastan claramente con aquellos que antaño representaban la visión normativa de un sujeto cartesiano autónomo. ${ }^{15}$

La desmesurada dependencia en la tecnociencia y la estrecha y ambigua relación entre el individuo y la máquina son signos elocuentes de una sociedad poshumana en la que la división binaria orgánico/artificial, "once so sacred to humanism" (Badmington 88), no sólo es problemática, sino también constantemente cuestionada en la ficción. De hecho, la deconstrucción de esta división binaria se manifiesta de manera clara si se analiza con detalle la interacción entre la líder Báez y su máquina personalizada MM. El perfil psicológico maquinal de Báez contrasta con una imagen bastante humanizada de la inteligencia artificial. Es decir, MM despliega por momentos dosis de buen humor, perspicacia o intuición, al punto de que ella misma cuestiona de manera consciente su propia artificialidad. Al mismo tiempo, MM pone en entredicho el grado de humanidad de su dueña Báez: "Tienes todo lo malo de los humanos [...] y nada de lo bueno" (9). Aunque Báez tampoco obvia la artificialidad de MM, el evidente grado de humanidad que despliega la máquina, pone en entredicho su admitida condición artificial: "tenía que reconocer que al hablar con

15 Partiendo de la analogía del "niño burbuja", en "Prophylaxis and Virulence" Jean Baudrillard advierte del peligro que supone para el individuo poshumano y su configuración, una total dependencia en la tecnología. 
ella, por mucho que fuera un montón de alambres y chips, se había puesto de mejor humor" (51). En relación al nivel de confusión y ambigüedad que provoca la división binaria orgánico-artificial Dona Haraway comenta:

Late twentieth century machines have made thoroughly ambiguous the difference between natural and artificial, mind and body, self-developing and externally designed, and many other distinctions that used to apply to organisms and machines. Our machines are disturbingly lively, and we ourselves frighteningly inert. (152) ${ }^{16}$

Cabe puntualizar que el lado más humanizado de $M M$ no radica en su capacidad para generar y procesar información con suma eficacia, sino que se ratifica a través de sus relaciones interpersonales con su dueña. Es cierto que muchos de los diálogos de tono serio y trascendental desvelan su naturaleza "mecánica" (51), pero también ilustran su lado más "humano". Destacan, sin ir más lejos, los diálogos de tono jocoso donde la oralidad y el uso de un lenguaje conversacional juvenil, insultante y contestatario, no sólo confirman la presencia del discurso santo y seña de la narrativa de la generación $X$, sino que nos revela un ente capaz de manejar varios registros lingüísticos con un grado de improvisación innatos en el ser humano.

En cuanto a la faceta emotiva se refiere, la máquina, como afirmaba más arriba Haraway, despliega signos vitales que se contraponen al comportamiento apático de un sujeto cada vez desligado de su naturaleza humana. Así vemos como Báez es glacial e insensible, mientras que MM se muestra "neurótica" o "mohína" (90), y constantemente "herida en su orgullo" (10). Báez no es ajena a este comportamiento y reconoce de manera autoconsciente la inversión identitaria de roles dentro de esta sociedad poshumana en la que se encuentra inmersa: "Las máquinas parecían personas y las personas parecían máquinas" (90). Esta idea sobre la ambigüedad en el comportamiento del sujeto y el grado de protagonismo que adquieren los organismos cibernéticos en Planeta hembra, se potencia aún más cuando la máquina cumple funciones que substancialmente moldean la condición vital del sujeto poshumano, huérfano al fin y al cabo, al ser gestado por "máquinas perfectas, que hacen las veces de útero" (120).

16 El premio Loebner se entrega cada año a científicos expertos en el campo de la inteligencia artificial que diseñan programas informáticos capaces de pasar el test de Turing. En 2014, por primera vez en la historia de este premio, un programa informático logró supuestamente superar el test de Turing al convencer al 33\% del jurado de que Eugene Goostman - un niño ucraniano de 13 años - era una persona, cuando en realidad se trataba de una inteligencia artificial. Para una historia completa relacionada con los parámetros del test de Turing y el programa informático Eugene Goostman, véase http://www.bbc.com/news/technology-27762088. 
En la novela esta imagen materna artificial se trata con bastante ironía, al ser MM la encargada de recordar a su jefa la necesidad de comer bien, de cuidar su colesterol y hacerse un chequeo urgente al descubrir que existe "una proteína preocupante en la orina" de Báez (51). ${ }^{17}$ El grado de protagonismo de la máquina en la vida del ser humano y el control que ejerce sobre el sujeto, provoca en la ficción momentos tensos que exteriorizan en clave irónica la ubicuidad opresiva de la inteligencia artificial: "Malditas máquinas. Estaban por todas partes. Midiéndolo todo. Analizándolo todo. Sacando conclusiones. Dando consejitos. Menuda plaga" (89). No obstante, resulta bastante irónico que esta visión paródica de la sociedad poshumana que refleja la novela de Bustelo, funciona a nivel extra-textual para plantear críticamente el grado de dependencia de una sociedad contemporánea, constantemente enganchada y moldeada por completo por un progreso tecnocientífico desenfrenado.

El universo distópico que plantea Planeta Hembra se basa en fundamentos tecnocientíficos más o menos probados para mantener la verosimilitud de lo narrado. Sin embargo, existen lagunas a la hora de exponer la configuración identitaria de algunos de los organismos cibernéticos, en especial la identidad de $M M$. El lector descubre que esta máquina es "custom-made" (50 cursiva de la autora), pero la capacidad de la misma para improvisar, evolucionar cognitivamente, y por ende, "sentir", es ante todo una incógnita. En este sentido, la ficción no llega nunca a confeccionar una visión convincente para explicar la naturaleza de este organismo cyborg. Esta carencia la suscita sin duda la falta de una base científica plausible, algo que en la ficción potenciaría su ontología como ente inteligente y enriquecería aún más el tono paródico que la autora propone dentro de la novela. En otras palabras, la configuración de este organismo cibernético podría vislumbrarse atendiendo por ejemplo a ciertas predicciones científicas como las que propone el investigador Hans Moravec, ${ }^{18}$ para quien la posibilidad de descargar la conciencia humana en un soporte artificial no es para nada irrazonable. ${ }^{19}$ Asimismo, este organismo cibernético tendría

17 En "Prophylaxis and Virulence", Jean Baudrillard afirma: "The growing cerebrality of machines must logically be expected to occasion a technological purification of bodies. Inasmuch as bodies are less and less able to count on their own antibodies, they are more and more in need of protection from outside. An artificial sterilization of all environments must compensate for faltering internal immunological defenses" (34).

18 Véase Mind Children: The Future of Robot and Human Intelligence (1988).

19 Katherine Hayles cuestiona esta posibilidad de separar la mente del cuerpo: "how could anyone think that consciousness in an entirely medium would remain unchanged, as if it had no connection with embodiment?" (1). 
una mayor credibilidad narrativa si se concibiera como un complejo sistema en el que se reproducen fenómenos biológicos en soportes artificiales, como el que concibe el biólogo evolucionista Thomas Ray, artífice del proyecto de vida artificial Tierra. ${ }^{20}$

Este sistema de vida artificial permite concebir "computer simulations of elementary organisms to see how they grow, reproduce and evolve through 'genetic mutations' that have been written into the software program" (Graham 3). El proyecto Tierra, en el que existen programas de software que llegan a copiar comportamientos innatos de los seres vivos, podría parecer descabellado, incluso exclusivo del entorno de la ciencia ficción literaria y cinemática. No obstante, como apunta Hayles, el principio organizador que permite imaginar estas simulaciones en los programas de software, is bridged largely through narratives that map the programs into evolutionary scenarios traditionally associated with the behavior of living creatures. The narratives translate the operations of computer codes into biological analogues that make sense of the program logic. (225)

Como le ocurre a MM, el sistema Tierra está conectado a Internet, de esta manera el proceso evolutivo no está confinado a la máquina, sino en libertad dentro de lo que podría concebirse como un ecosistema digital global en continua evolución.

\section{POLITIZACIÓN Y MONOPOLIO BIOTECNOLÓGICO Y TECNOCIENTÍFICO}

Lejos de ser una simple postura dialéctica entre sexos, el monopolio biotecnológico y tecnocientífico que ostenta la mujer en Planeta hembra tiene una clara connotación reivindicativa que se extiende incluso más allá de la ficción. De hecho, críticos como Gill Kirkup señalan que históricamente la mujer ha ocupado un lugar secundario, casi testimonial en la investigación y control tecnocientífico: "The systems and artifacts produced by technoscience were seen as providing the material foundations for gender inequality" (XIII). Si bien es cierto que Planeta hembra debe leerse en

20 La investigación en torno a la vida artificial se divide en tres campos: "Wetware is the attempt to create artificial biological life through such techniques as building components of unicellular organism in the test tubes. Hardware is the construction of robots and other embodied life forms. Software is the creation of computer programs instantiating emergent or evolutionary processes" (Hayles 225). 
clave paródica feminista, en cierto modo la ficción reproduce de manera implícita el deseo femenino de reclamar para la mujer un espacio en el campo de la investigación científica y tecnológica, del que ha sido excluida históricamente.

A nivel extra-textual, algunos indicios apuntan a que dentro de una sociedad poshumana futura el protagonismo femenino en torno al control tecnocientífico podría llegar a equiparse al que ostenta el hombre, especialmente si se tiene en cuenta que las diferencias genéricas son cada vez menores en un presente histórico: "The technosciences of the late twentieth and early twenty-first century are proposed as having a different impact and set of possibilities for gender relations and for women" (Kirkup XIV-XIV). En esta misma línea de pensamiento se ha posicionado con anterioridad Dona Haraway, para quien la identidad cyborg, "a creature in a post-gender world" (150), hay que concebirla dentro de un paradigma genérico más equitativo.

Uno de los signos vitales de la poshumanidad se manifiesta precisamente en el control desmesurado o una manipulación genética excesiva ejercida por y sobre el ser humano. En Cyborg City, Chris Hables Gray analiza en profundidad el concepto de participatory evolution para referirse al modo en que la dinámica de la evolución natural es suplantada por una evolución artificial, controlada parcialmente por el ser humano: "Artificial evolution is not just the conscious breeding of farm animals that Darwin discussed; it now includes the direct modification of human bodies and genes" (1 1). Para el autor, los motivos principales que mueven la evolución artificial son principalmente políticos y será precisamente la política la que establezca los valores básicos de una poshumanidad (11). En este sentido, Planeta hembra parodia la moralidad de una política feminista radical que mueve los hilos de la tecnociencia para ejercer el control político, genérico y demográfico del planeta:

Se mantienen los rasgos biológicos propios de cada etnia, pero el número de individuos pertenecientes a cada una de ellas está pre-establecido [...] Dado el alto coste [...] la individualización y mejora genética sólo se llevan a cabo en el caso de las Hembras Reales. El resto de los seres humanos, Hembras No Reales y Hombres, serían una repetición ad eternum de personas que ya han existido. (128 cursiva de la autora)

Hables Gray y otros autores como Francis Fukuyama, que hablan de las implicaciones éticas de una supuesta sociedad poshumana, advierten del peligro que supone en el futuro politizar el acceso y control de la tecnología y la ciencia. Elaine Graham comenta al respecto que al concebir en el futuro una sociedad tecnocrática, producto de la era digital y la bio- 
tecnología, se evaden cuestiones fundamentales tales como "[the] access to technoscientific resources and opportunities" (8). En el caso de Planeta hembra la carencia de una política trasparente en relación a la explotación de las nuevas tecnologías provoca un uso indiscriminado de la manipulación genética, lo que precipita la configuración de un sujeto poshumano replicado, esclavo del deseo egoísta y caprichoso de una élite tecnocrática feminista despiadada.

\section{ManipulaCión tecnO-SeXUal y paradigma Genérico-SeXUal alternativo}

En la novela de Bustelo, los avances tecnocientíficos contribuyen al desarrollo de una experiencia sexual artificial desmesurada. Sin embargo, como en cualquier sistema de gobierno autoritario, la experiencia sexual artificial no escapa a la politización y el monopolio de un sector reducido de la élite femenina. En Planeta, la experimentación sexual a través de la tecnología, podría interpretarse en clave feminista como la violación de una identidad femenina normativa que está delimitada por una capacidad "in mothering and its metaphoric extensions" (Haraway 180). En este sentido, las líderes cyborg de Planeta reformulan de manera drástica su interacción tecnocientífica para explorar "aspect[s] of sex and sexual embodiment" (ibíd. 180), transgrediendo así una conducta heterosexual sistemática. Llama la atención que en la novela la forma de superar la construcción genérica y sexual dominante dentro de un paradigma humanista - en el que " $t]$ he systems and artifacts produced by technoscience were seen as providing the material foundations for gender inequality" (Kirkup XIII) -, radica precisamente en experimentar "intense pleasure in machines" (Haraway 180). En su búsqueda de placer y de una identidad sexual libre de la influencia patriarcal, el feminismo radical de Planeta origina de manera inversa una tecnocracia que altera las relaciones de género y relega al hombre a una marginalidad política, social y tecnocientífica. Sin embargo, la ubicuidad de un patriarcado no deja nunca de estar presente en la ficción y parece refrendarse al final de la novela al consumarse la relación heterosexual entre Báez y Graf.

El mundo distópico de Planeta hembra brinda al lector la posibilidad de concebir una versión de una identidad poshumana femenina completamente enganchada al placer tecnológico y a la continua experimentación con y en el cuerpo femenino. Un ejemplo muy ilustrativo de este fenómeno dentro de la ficción es la ingesta habitual del virtux, un fármaco que "ofrece excelentes resultados con el virtual sexual" (80). El virtux y otras posibilidades de experimentación sexual tecnocientíficas posibilitan "ways of thinking [an acting] that look beyond the phallocentric, or male-centered, subject of hu- 
manism" (Toffoletti 10). Por desgracia, la experimentación sexual a través de la tecnología, especialmente en el caso de las líderes, condiciona de manera negativa la configuración de una identidad femenina. En la ficción dicha identidad se presenta sarcásticamente mediante dos estereotipos de mujeres: "Recelosas paranoides y exhibicionistas sicóticas" (27). Es evidente que en varias instancias la novela aborda con sarcasmo la identidad cyborg femenina y las consecuencias negativas de un feminismo lésbico radical, parodiando para ello el subgénero literario de la ciencia ficción feminista. Sin embargo, sería erróneo obviar las posibilidades e implicaciones que ofrece la tecnociencia a la hora de configurar una identidad femenina que busca liberarse de restricciones genéricas patriarcales.

Lo cierto es que la convergencia entre lo biológico y lo artificial permite hoy en día concebir la materialización de un paradigma poshumano que ya ha generado, entre otras cosas, el modo de repensar la identidad de género más allá de una concepción humanista. En la ficción, las líderes del partido XX intentan generar dicha identidad a través del uso desmedido de tecnologías diseñadas para la experimentación sexual en y con el cuerpo femenino. Y si bien es verdad que dicha experimentación sexual tecnocientífica resulta un tanto improbable en un presente histórico, el principio tecnocientífico que la viabiliza no es para nada inverosímil. Sin ir más lejos, descubrimientos tecnocientíficos recientes como el del doctor Stuart Meloy, creador del orgasmatron, confirman esta posibilidad. Este dispositivo con fines terapéuticos "involves the insertion of a remote controlled device into a woman's spinal column which, when activated, electronically stimulates the body's nervous system, specifically those neural pathways leading to coital nirvana" (Toffoletti 9). ${ }^{21}$ En su concepción del cyborg, Katherine Hayles señalaba que "[c]entral to the construction of the cyborg are informational pathways connecting the organic body to its prosthetic extensions" (2). La aplicación del orgasmatron al cuerpo femenino no debería vislumbrarse como algo meramente anecdótico de la convergencia entre lo artificial y lo biológico, sino más bien como un modelo muy sugerente para ilustrar cómo avances biotecnológicos y tecnocientíficos llegan a plantear nuevas vías para cuestionar más que nunca la clasificación normativa de un sujeto femenino contemporáneo.

21 El orgasmatron hace un guiño evidente al tubo electromecánico que en los años setenta hiciera célebre Woody Allen en su película "Sleeper" (United Artists, 1973). Para un informe detallado del descubrimiento y funcionamiento del Orgasmatron, véase "Call him doctor 'Orgasmatron." http://www.latimes.com/health/la-he-orside 1 feb 1 1-story.html. 
En pocas palabras, la unión biológico/artificial que se forja al converger el orgasmatron con el cuerpo femenino, podría considerarse como todo un hito para repensar una concepción feminista de la identidad cyborg en un presente histórico cada vez más poshumano. De hecho, si pensamos por un momento en clave feminista, es evidente que esta simbiosis no sólo contradice considerablemente un discurso humanista en torno a las oposiciones binarias, sino que además genera una imagen provocativa de otra especie cyborg: una identidad que invita a considerar la posibilidad de seguir imaginando un sujeto femenino poshumano que añora "[an] utopian dream of hope for a monstrous world without gender" (Haraway 181). ${ }^{22}$

\section{Biblografía}

ALTISENT, Marta E. "El mundo antitético de Planeta hembra de Gabriela Bustelo." La pluralidad narrativa: escritores españoles contemporáneos (1984-2004). Eds. Ángeles Encinar and Kathleen M. Glenn. Madrid: Biblioteca Nueva, 2005. 89-106. Impreso.

BADMINGTON, Neil. Alien Chic: Posthumanism and the Other Within. London and New York: Routledge, 2004. Impreso.

BAUDRILLARD, Jean. "Prophylaxis and Virulence." Posthumanism. Ed. Neil Badmington. New York: Palgrave, 2000. 34-41. Impreso.

BUSTELO, Gabriela. Planeta hembra. Barcelona: RBA, 2001. Impreso.

Graham, Elaine L. Representations of the Post/Human: Monsters, Aliens and Others in Popular Culture. New Brunswick, New Jersey: Rutgers UP, 2002. Impreso.

HABLES GRAY, Chris. Cyborg Citizen: Politics in the Posthuman Age. New York and London: Routledge, 2001. Impreso.

HALBERSTAM, Judith and Ira Livingston "Introduction." Posthuman Bodies. Eds. Judith Halberstam and Ira Livingston. Indianapolis: Indiana UP, 1995. 1-19. Impreso.

HARAWAY, Donna J. "A Cyborg Manifesto: Science, Technology, and Socialist-Feminism in the Late Twentieth Century." Simians, Cyborgs and Women: The Reinvention of Nature. New York: Routledge, 1991. 149-181. Impreso.

22 Críticas feministas como Paula Rabinowitz se muestran escépticas a la hora de poder concebir la idea de una mujer poshumana, desligada por completo de una ideología humanista binaria. Rabinowitz arguye que "the posthuman body is still saturated with the stories of humanity that circulate around it; it speaks through a language straddling the borders between health/ sickness, male/female, real/imaginary (98). 
HASSAN, Ihab. "Prometheus as Performer: Toward a Postmodern Culture?" Performance in Postmodern Culture. Eds. Michel Benamou and Charles Caramello. Milwaukee: Centre for Twentieth Century Studies, University of Wisconsin-Milwaukee, 1977. 201-217. Impreso.

HAYLES, Katherine. How We Became Posthuman: Virtual Bodies in Cybernetics, Literature, and Informatics. Chicago: University of Chicago Press, 1999. Impreso.

KIRKUP, Gill et al. The Gendered Cyborg: A Reader. London and New York: Routledge, 2000. Impreso.

PEPPERELL, Robert. The Post-Human Condition: Consciousness Beyond the Brain. Bristol, UK: Intellect Books, 2003. Impreso.

RABINOWITZ, Paula. "Soft Fictions and Intimate Documents: Can Feminism Be Posthuman?"

Posthuman Bodies. Eds. Judith Halberstam and Ira Livingston, Indianapolis: Indiana UP, 1995. 97-1 12. Impreso.

TOFFOLETTI, Kim. Cyborgs and Barbie Dolls: Feminism, Popular Culture and the Posthuman Body. London and New York: I.B. Tauris, 2007. Impreso. 